\title{
Research Article \\ Effects of Time Delay and Noise on Asymptotic Stability in Human Quiet Standing Model
}

\author{
Caihong Wang and Jian Xu \\ School of Aerospace Engineering and Applied Mechanics, Tongji University, Shanghai 200092, China \\ Correspondence should be addressed to Jian Xu, xujian@mail.tongji.edu.cn
}

Received 25 December 2009; Revised 12 May 2010; Accepted 2 September 2010

Academic Editor: Carlo Cattani

Copyright (C 2010 C. Wang and J. Xu. This is an open access article distributed under the Creative Commons Attribution License, which permits unrestricted use, distribution, and reproduction in any medium, provided the original work is properly cited.

\begin{abstract}
A human quiet standing stability is discussed in this paper. The model under consideration is proposed to be a delayed differential equation (DDE) with multiplicative white noise perturbation. The method of the center manifold is generalized to reduce a delayed differential equation to a two-dimensional ordinary differential equation, to study delay-induced instability or Hopf bifurcation. Then, the stochastic average method is employed to obtain the Itô equation. Thus, the top Lyapunov exponent is calculated and the necessary and sufficient condition of the asymptotic stability in views of probability one is obtained. The results show that the exponent is related to not only the strength of noise but also the delay, namely, the reaction speed of brain. The effect of the strength of noise on the human quiet standing losing stability is weak for a small delay. With the delay increasing, such effect becomes stronger and stronger. A small change in the strength of noise may destabilize the quiet standing for a large delay. It implies that a person with slow reaction is easy to lose the stability of his/her quiet standing.
\end{abstract}

\section{Introduction}

The human quiet standing model is complex neuromuscular control biological system with time delay. The time delay reflects finite transmission related to the transport or processing of matter, energy, and signals through the systems [1,2]. Noise is an immanent property in biological systems, and such stochasticity may arise from muscle contractions as well as imperfections, and nervous system operates under the very noisy environment in the balance control system. Previous experimental studies have shown that the movement of the pressure center during quiet standing is stochastic and obeys a correlated random walk $[3,4]$. For healthy people, balancing body stability is an easy task. However, older people or some patients often find walking difficult and need to put more effort in quiet standing. To help these groups, the principles making quiet standing must be discovered firstly. As the gravitational force destabilizes the inverted-pendulum-like skeletal system, 
the study of human balance stability is extremely essential. However, serious difficulties will be encountered because of the combination of delay and stochastic processes when the qualitative stochastic differential delay system is investigated in human quiet standing.

In recent years, many researchers have studied the postural control system in quiet standing [5-21]. Experimentally, human quiet standing has most often been investigated in terms of the center of pressure (COP) trajectories $[3,10]$. The time-delayed model is studied by the measurements of the transfer function which is based on statistical quantities such as the root mean square of the COP trajectory [9, 11]. In 2004, Verdaasdonk et al. [12] have computed the branches of fold and Hopf bifurcation by numerical bifurcation analysis of the inverted pendulum human model indicated that the period of the stable limit cycles, emerging beyond the Hopf branch, will increase with increasing time delay. Bottaro et al. [13] have indicated that the control in postural sway during quiet standing is intermittent, but not continuous. The effects of delay for the human reaction during balancing system without stochastic perturbation have been studied in [5-7]. In 2002, based on the investigation of a delayed random walk with an unstable fixed point, it has been observed that the random walker with delay moves away from the unstable fixed point more slowly than with the absence of delay [15]. The deterministic Hopf bifurcation has been qualitatively discussed with the help of stochastic delayed differential equations (DDEs) by Yao et al., but the stochastic effect was only made by numerical analysis [18]. In [19], a novel modeling approach to such two-feedback posture control has been proposed using a system of stochastic delay differential equations with two delays and noise as well as a drifting fixed point meant to represent the slower fluctuation of the COM. Effects of time delay on the dynamics behavior of the human quiet standing systems are a subject of many experimental studies, while the study on simultaneous consideration of time delay and stochastic excitation is very limited. Most of researches about the time-delay human standing model still with stochastic perturbation are studied by numerical simulation.

In this paper, we consider the single inverted pendulum model proposed by Eurich et al. $[9,11,18,19]$. The single inverted pendulum with an antagonistic muscle pair represents a person who tries to maintain an upright position by flexing and extending the ankles. Influence of delay and noise on the Hopf bifurcation and asymptotic stability will be analyzed theoretically. However, it becomes infinite-dimensional problem due to considering the time delay in quiet standing system, which increases work difficulty. In stochastic systems, theoretically studied methods about bifurcation and stability are rare. Existing research methods are limited, such as the stability of a linearly controlled system with time delay subjected to Gaussian white noise which has been investigated using the top Lyapunov exponent calculated from Monte Carlo simulation by Grigoriu [22]. The asymptotic Lyapunov stability with probability one for quasi-integrable and nonresonant Hamiltonian systems with time-delayed feedback control has been studied in terms of the stochastic average method [23]. Asymptotic techniques, such as Taylor series expansion, integral averaging method, Fourier series, and perturbation methods, are often used under the assumption of small delays. However, these methods will be invalid for a large delay. Therefore, we will reduce a stochastic DDE to a stochastic ordinary differential equation (ODE) on the center manifold by adopting the center manifold method which has been proved by Arnold and Boxler [24]. The existence of the stochastic center manifold has been also proved in [24-26]. If a stochastic DDE is reduced to a stochastic ODE, then this ODE on the centre manifold may be converted into amplitude and phase relations and corresponding scalar bifurcation equations of amplitude may be obtained in terms of the integral averaging method [27]. 
The paper is organized as follows. In Section 2, the human quiet standing model is introduced and the conditions of Hopf bifurcation are computed in order to obtain regions of stability and instability. Section 3 is devoted to effects of time delay and noise on the asymptotic stability of the human dynamics with stochastic perturbation. Section 4 analyzes the application to balance. Finally, we draw conclusions.

\section{The Model and Linearized Analysis}

In this paper, we use a simplified single inverted pendulum model for the human in quiet standing with stochastic perturbation $[6,8,16]$ :

$$
I \ddot{\theta}+\gamma \dot{\theta}-m g L \sin \theta=\tilde{f}(\theta(t-\tau))+\tilde{c} \eta(t) \theta(t),
$$

where $I$ represents the moment of inertia of human body around the ankle, $\theta$ the tilt angle, $g$ the gravity acceleration, $\mathrm{m}$ the body mass, $L$ the distance from the ankle joint to the body COM (Center of Mass), $\gamma$ the damping coefficient, $f(x(t))$ the postural sway feedback function, $\tau$ the time delay, and $\eta(t)$ a stochastic process of zero mean value Gauss white noise.

Let $q=L \sin \theta$, where $x$ is the transverse displacement of the gravity center. Then (2.1) can be rewritten as follows:

$$
\ddot{q}=\frac{m g L}{I} q-\frac{r}{I} \dot{q}+f+\widetilde{\widetilde{b}} \eta(t) q(t)
$$

Then (2.2) can be written as

$$
\dot{x}(t)=a x(t)+b f(x(t-\tau))+c \eta(t) x(t),
$$

where

$$
x(t)=q+d \dot{q}, \quad a=\frac{m g L}{I}\left(\frac{r}{I}+\sqrt{\frac{r^{2}}{I^{2}}+4 \frac{m g L}{I}}\right)>0, \quad d=\frac{r}{I}+\sqrt{\frac{r^{2}}{I^{2}}+4 \frac{m g L}{I}},
$$

and $b<0$ is the feedback coefficient. The feedback function $f(x(t))$ should be in the form of a smoothed on-off switch at some delayed time $\tau_{0}$ for proprioception at the ankles, so we take $f(x(t-\tau))=\tanh [x(t-\tau)]$ in this paper. Since $\tanh [x(t)]=x(t)-(1 / 3) x^{3}(t)+o\left(x^{4}\right)$ by the Taylor expansion, then (2.2) becomes

$$
\dot{x}(t)=a x(t)+b x(t-\tau)-\frac{b}{3} x^{3}(t-\tau)+c \eta(t) x(t)+o\left(x^{4}\right) .
$$

The linear part of (2.2) is given by

$$
\dot{x}(t)=a x(t)+b x(t-\tau) .
$$




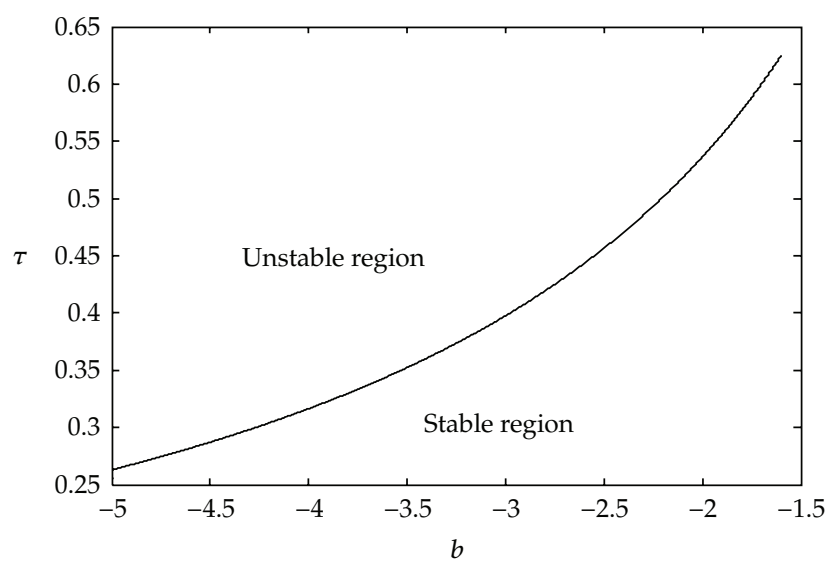

Figure 1: The critical values of $\tau_{0}$ under Hopf bifurcation.

Trial exponential solutions of the form $x(t)=e^{\lambda t}, x(t-\tau)=e^{\lambda}(t-\tau)$ yield the transcendental characteristic equation

$$
\Delta(\lambda, \tau)=a+b e^{-\lambda \tau}-\lambda=0
$$

The stability of system (2.3) depends on the real parts sign of the roots in (2.5). Let $\lambda=v+i w$ be a root of the characteristic equation (2.7), where $v$ and $w$ take real values. Substituting this expression into (2.7), and equating the real and imaginary parts to zero, we have a pair of algebraic equations

$$
v=a+b e^{-v \tau} \cos w \tau, \quad w=-b e^{-v \tau} \sin w \tau .
$$

The transversality condition by the implicit function theorem is

$$
\operatorname{Re}\left(\frac{d v}{d \tau}\right)=b e^{-v \tau}\left(-\frac{\partial v}{\partial \tau} \tau-v\right) \cos w \tau-w b e^{-v \tau} \sin w \tau \neq 0 .
$$

As a necessary condition for Hopf bifurcation, we have to put $v=0$ in (2.8). The following algebraic equations are obtained:

$$
w= \pm \sqrt{b^{2}-a^{2}}, \quad a+b \cos \tau \sqrt{b^{2}-a^{2}}=0 .
$$

Varying the coefficient $a$ and keeping $a=1.6$, one can draw the parametric place $(b, \tau)$ as Figure 1, namely, stability region diagram.

\section{Stability of the Hopf Bifurcation}

We have obtained the conditions that system (2.3) undergoes a Hopf bifurcation at the equilibrium point $(0,0)$ when bifurcation parameter $\tau$ passes through the critical value. 
In this section, the bifurcating stability of system (2.3) at $\tau_{0}$ will be presented by employing the central manifold reduction and stochastic average method.

\subsection{The Stochastic ODE}

In this section, we derive the center manifold of the nonlinear stochastic ODEs which reduce the DDE with infinite dimension to ODE with two dimension. To this end, let $t=\tau s, u(t)=$ $x(t \tau)$. By rescaling the time to normalize the delay, system (2.5) is equivalent to the following equation

$$
\dot{u}(t)=\tau\left(a u(t)+b u(t-1)-\frac{b}{3} u^{3}(t-1)+c u(t) \eta(t)\right)+o\left(u^{4}(t-1)\right) .
$$

We choose the delay $\tau$ as bifurcation parameter, which is subject to a small change $\varepsilon \mu$ from its critical value $\tau_{0}$, namely, $\tau=\tau_{0}+\varepsilon \mu$. The values of $u, c$ have been rescaled, and we obtain $u \rightarrow \varepsilon^{1 / 2} u, \quad c \rightarrow \varepsilon^{1 / 2} c, 0<\varepsilon \ll 1$. Then (3.1) can be rewritten as follows:

$$
\dot{u}_{t}(0)=\tau_{0}\left(a u_{t}(0)+b u_{t}(-1)\right)+\varepsilon \mu\left(a u_{t}(0)+b u_{t}(-1)\right)+g\left(\mu, u_{t}(-1)\right),
$$

where $u_{t}(\theta)=u(t+\theta), \theta \in[-\tau, 0]$.

The linear part of (3.2) is given by

$$
\dot{u}(t)=\tau_{0}(a u(t)+b u(t-1))+\varepsilon \mu(a u(t)+b u(t-1)) .
$$

Since $0<\varepsilon \ll 1$, the nonlinear part is given by

$$
g\left(\mu, u_{t}\right)=-\frac{b}{3} \tau u_{t}^{3}(-1)+\varepsilon^{-1 / 2} \tau c \eta(t) u_{t}(0)
$$

Let $L_{\mu}: C[-1,0] \rightarrow R$, which is a one-parameter family of bounded continuous linear operator. Then

$$
L(0) u_{t}=\tau_{0}\left(a u_{t}(0)+b u_{t}(-1)\right), \quad L(\mu) u_{t}=\mu\left(a u_{t}(0)+b u_{t}(-1)\right) .
$$

By Riesz representation theorem, there exists a bounded function $\zeta(\theta, \mu)$ in $[-1,0] \rightarrow R$ for any $\phi \in C[-1,0]$, such that

$$
L(0) \phi=\int_{-1}^{0}[d \zeta(\theta)] \phi(\theta), \quad L(\mu) \phi=\int_{-1}^{0}[d \zeta(\theta, \mu)] \phi(\theta)
$$


where

$$
\begin{aligned}
& \zeta(\theta, \mu)=\tau_{0}(a \delta(\theta)+b \delta(\theta+1))(\delta(\theta) \text { is Dirac function), } \\
& A(0) \phi= \begin{cases}\frac{d \phi(\theta)}{d \theta} & \text { for } \theta \in[-1,0), \\
L(0) \phi & \text { for } \theta=0,\end{cases} \\
& A(\mu) \phi= \begin{cases}\frac{d \phi(\theta)}{d \theta} & \text { for } \theta \in[-1,0), \\
L(\mu) \phi & \text { for } \theta=0,\end{cases} \\
& D \phi= \begin{cases}0 & \text { for } \theta \in[-1,0), \\
g(t, \phi) & \text { for } \theta=0 .\end{cases}
\end{aligned}
$$

In order to study Hopf bifurcation problem conveniently, we can rewrite (3.2) as a function differential equation (FDE)

$$
\dot{u}_{t}=A(0) u_{t}+\varepsilon A(\mu) u_{t}+\varepsilon D u_{t}
$$

The adjoint equation of (3.3) is given by

$$
\dot{w}(\hat{t})=-\tau_{0}(a w(\widehat{t})+b w(\widehat{t}+1)) \quad \widehat{C}:=([0,1], R)
$$

The corresponding operator $A^{*}$ of $A$ is defined by

$$
A^{*}(\mu) \psi= \begin{cases}-\frac{d \psi(s)}{d s}, & s \in(0,1] \\ \int_{-1}^{0}\left[d \zeta^{T}(s, \mu)\right] \psi(-s), & s=0\end{cases}
$$

and the bilinear relation

$$
\begin{array}{r}
\left(\psi_{j}(s), \phi_{k}(\theta)\right)=\left(\psi_{j}(0), \phi_{k}(0)\right)+\tau_{0} b \int_{-1}^{0} \psi_{j}(\xi+1) \phi_{k}(\xi) d \xi, \\
\psi_{j}(s) \in \widehat{C}, \quad \phi_{k}(\theta) \in C, \quad j, k=1,2,
\end{array}
$$

where $\widehat{C}$ is the dual space of $C, \Phi \in C, \Psi \in \widehat{C}$. Hale and his colleagues have shown that there exist two disjoint subspaces $P, Q$ as $C=P \oplus Q$. From the Hopf bifurcation conditions given in the previous section, we know the subspace $P$ is the eigenspace corresponding to the eigenvalues $\pm w i$ of $\Delta(\lambda, \tau)=0$ at Hopf bifurcation, and $Q$ is the infinite-dimensional complementary subspace associated with the remaining eigenvalues $\Delta(\lambda, \tau)=0$. For the particular eigenvalues $\lambda_{1,2}= \pm w i$, we have $\phi(\theta)=\Phi(\theta) \beta \in C(-1 \leq \theta \leq 0)$ and $\psi(s)=$ $\Psi(s) \widehat{\beta} \in \widehat{C}(0 \leq s \leq 1)$, where the values $\Phi(\theta)=\left[\phi_{1}(\theta), \phi_{2}(\theta)\right]$ and $\Psi(s)=\left[\psi_{1}(s), \psi_{2}(s)\right]^{\mathrm{T}}$. 
We define the elements of the initial continuous function $\phi(\theta) \in C$, projections $\phi^{P}(\theta), \phi^{Q}(\theta)$ onto the center, and stable subspaces $P, Q \in C$. Through the initial function $\phi(\theta) \in C$, suppose $x_{t}(\phi(\theta), \mu, \varepsilon) \in C$ be the unique solution of (2.1). Then we have the representations

$$
u_{t}(\phi(\theta), \mu, \varepsilon)=u_{t}^{P}(\phi(\theta), \mu, \varepsilon)+u_{t}^{Q}(\phi(\theta), \mu, \varepsilon)
$$

and $\phi(\theta)=\phi^{P}(\theta)+\phi^{Q}(\theta)$, where $x_{t}^{P}(\phi(\theta), \mu, \varepsilon), \quad \phi^{P}(\theta) \in P$ and $x_{t}^{Q}(\phi(\theta), \mu, \varepsilon), \phi^{Q}(\theta) \in Q$. Making a change of variables $x_{t}^{P}(\theta)=\Phi(\theta) y(t)+x_{t}^{Q}(\theta)$ with $y(t)=(\bar{\Psi}(s), \phi(\theta)) \in R^{2}$, then the center manifold stochastic ODEs of the generalized eigenspace $P \in C$ can be obtained:

$$
\dot{y}(t)=B y(t)+\varepsilon \bar{\Psi}(0) g(\mu, y(t))
$$

where the values $B$ and $\bar{\Psi}(0)$ are obtained in Appendix A.

We obtain

$$
\begin{gathered}
\dot{y}_{1}(t)=-w y_{2}-\varepsilon p_{1}\left(a \mu y_{1}(t)+b \mu\left(y_{1}(t) \cos w \theta+y_{2}(t) \sin w \theta\right)\right. \\
\left.-\frac{1}{3} b \tau\left(y_{1}(t) \cos w \theta-y_{2}(t) \sin w \theta\right)^{3}+\varepsilon^{-1 / 2} \tau c \eta(t) y_{1}(t)\right), \\
\dot{y}_{2}(t)=w y_{1}-\varepsilon p_{2}\left(a \mu y_{1}+b \mu\left(y_{1}(t) \cos w \theta+y_{2}(t) \sin w \theta\right)\right. \\
\left.-\frac{1}{3} b \tau\left(y_{1}(t) \cos w \theta+y_{2}(t) \sin w \theta\right)^{3}+\varepsilon^{-1 / 2} \tau c \eta(t) y_{1}(t)\right),
\end{gathered}
$$

where

$$
p_{1}=\frac{-\psi_{22}}{\psi_{11} \psi_{22}-\psi_{12}^{2}}, \quad p_{2}=\frac{\psi_{12}}{\psi_{11} \psi_{22}-\psi_{12}^{2}} .
$$

Carrying out a change of variables from $\left(y_{1}, y_{2}\right)$ to $\left(z_{1}, z_{2}\right)$, namely,

$$
z_{1}=y_{1}-\frac{p_{1}}{p_{2}} y_{2}, \quad z_{2}=y_{2}-\frac{p_{1}}{p_{2}} w y_{1} .
$$

we obtain expressions for $\left(y_{1}, y_{2}\right)$

$$
\begin{aligned}
& y_{1}=\frac{p_{2}^{2}}{p_{2}^{2}+p_{1}^{2} w^{2}}\left(z_{1}+\frac{p_{1}}{p_{2}} w z_{2}\right) \\
& y_{2}=\frac{p_{2}^{2}}{p_{2}^{2}+p_{1}^{2} w^{2}}\left(z_{2}-\frac{p_{1}}{p_{2}} w z_{1}\right) .
\end{aligned}
$$


Then the centre manifold stochastic ODEs (3.14) become

$$
\begin{aligned}
& \dot{z}_{1}(t)=-w z_{2}, \\
& \begin{aligned}
\dot{z}_{2}(t)=w z_{1}-\varepsilon \frac{p_{2}^{2}+p_{1}^{2} w}{p_{2}}( & -\frac{1}{3} b \tau\left(p_{11} z_{1}(t)+p_{12} z_{2}(t)\right)^{3} \\
& \left.+\mu\left(q_{11} z_{1}(t)+q_{12} z_{2}(t)\right)+\varepsilon^{-1 / 2} \tau c \eta(t)\left(g_{11} z_{1}(t)+g_{12} z_{2}(t)\right)\right),
\end{aligned}
\end{aligned}
$$

where

$$
\begin{gathered}
p_{11}=\frac{p_{2}^{2}}{p_{2}^{2}+p_{1}^{2} w^{2}}\left(\cos w-\frac{p_{1}}{p_{2}} w \sin w\right), \quad p_{12}=\frac{p_{2}^{2}}{p_{2}^{2}+p_{1}^{2} w^{2}}\left(\sin w+\frac{p_{1}}{p_{2}} w \cos w\right), \\
q_{11}=\frac{p_{2}^{2}}{p_{2}^{2}+p_{1}^{2} w^{2}}\left(a+b \cos w-\frac{b p_{1}}{p_{2}} w \sin w\right), \quad g_{11}=\frac{p_{2}^{2}}{p_{2}^{2}+p_{1}^{2} w^{2}} \\
q_{12}=\frac{p_{2}^{2}}{p_{2}^{2}+p_{1}^{2} w^{2}}\left(\frac{p_{1}}{p_{2}}(a+b w \cos w)+\sin w\right), \quad g_{12}=\frac{p_{2} p_{1} w}{p_{2}^{2}+p_{1}^{2} w^{2}} .
\end{gathered}
$$

Then, using the relations $z_{1}=\beta(t) \sin \Theta, z_{2}=-\beta(t) \sin \Theta$, and $\Theta=w t+\varphi(t)$, (3.18) is transformed to the following system with polar coordinates:

$$
\begin{aligned}
\dot{\beta}(t)=\varepsilon \frac{p_{2}^{2}+p_{1}^{2} w}{p_{2}^{2}} \beta( & -\frac{1}{3} b \beta^{2} \tau\left(p_{11} \sin \Theta-p_{12} \cos \Theta\right)^{3} \\
& \left.+\mu\left(q_{11} \sin \Theta-q_{12} \cos \Theta\right)+\varepsilon^{-1 / 2} \tau c \eta(t)\left(g_{11} \sin \Theta-g_{12} \cos \Theta\right)\right) \cos \Theta, \\
\dot{\varphi}(t)=-\varepsilon \frac{p_{2}^{2}+p_{1}^{2} w}{p_{2}^{2}}( & -\frac{1}{3} b \tau \beta^{2}\left(p_{11} \sin \Theta-p_{12} \cos \Theta\right)^{3} \\
& \left.+\mu\left(q_{11} \sin \Theta-q_{12} \cos \Theta\right)+\varepsilon^{-1 / 2} \tau c \eta(t)\left(g_{11} \sin \Theta-g_{12} \cos \Theta\right)\right) \sin \Theta .
\end{aligned}
$$

\subsection{The Maximum Lyapunov Exponent}

In the above subsection, we reduce the system to a two-dimensional ordinary differential equation (3.20). According to the Khasminskii limit theorem, we obtain the averaging Itô stochastic equations of (3.20) as follows:

$$
\begin{aligned}
d \beta(t)= & \frac{p_{2}^{2}+p_{1}^{2} w}{p_{2}^{2}} \varepsilon \beta\left(\frac{1}{8} b \tau\left(p_{11}^{2} p_{12}+p_{12}^{3}\right) \beta^{2}-\frac{1}{2} q_{12} \mu+\frac{p_{2}^{2}+p_{1}^{2} w}{4 p_{2}^{2}} \tau^{2}\left(g_{11}^{2}+g_{12}^{2}\right) c^{2}\right) d t \\
& +\varepsilon^{1 / 2} \frac{\sqrt{2\left(g_{11}^{2}+3 g_{12}^{2}\right)}\left(p_{2}^{2}+p_{1}^{2} w\right)}{4 p_{2}^{2}} \tau \beta c d w_{1},
\end{aligned}
$$




$$
\begin{aligned}
d \varphi(t)= & \varepsilon \frac{p_{2}^{2}+p_{1}^{2} w}{p_{2}^{2}}\left(\frac{1}{8} b \tau\left(p_{11}^{3}+p_{11} p_{12}^{2}\right) \beta^{2}-\frac{1}{2} q_{11} \mu\right) d t \\
& +\varepsilon^{1 / 2} \frac{\sqrt{2\left(3 g_{11}^{2}+g_{12}^{2}\right)}\left(p_{2}^{2}+p_{1}^{2} w\right)}{4 p_{2}^{2}} \tau c d w_{2}
\end{aligned}
$$

where $w_{1}, w_{2}$ are independent standard Wiener processes. The averaged amplitude and phase of (3.21a) and (3.21b) are uncoupled, so we study stability using the averaged amplitude equation (3.21a). To examine the stability of $\beta_{0}(t)$, let $\beta(t)=\beta_{0}(t)+r(t)$ and $\rho(t)=\ln r(t)$, where $r(t)$ represents a small variation around the stationary solution $\beta_{0}(t)$ when $d \beta(t)=0$, and using the stochastic differential rule to Itô, we will obtain the linearized Itô equation governing $r(t)$ and $\rho(t)$, namely,

$$
\begin{aligned}
d r(t)= & \frac{p_{2}^{2}+p_{1}^{2} w}{p_{2}^{2}} \varepsilon r\left(\frac{3}{8} b \tau\left(p_{11}^{2} p_{12}+p_{12}^{3}\right) \beta_{0}^{2}-\frac{1}{2} q_{12} \mu+\frac{p_{2}^{2}+p_{1}^{2} w}{4 p_{2}^{2}} \tau^{2}\left(g_{11}^{2}+g_{12}^{2}\right) c^{2}\right) d t \\
& +\varepsilon \frac{1}{2} \frac{\sqrt{2\left(g_{11}^{2}+3 g_{12}^{2}\right)}\left(p_{2}^{2}+p_{1}^{2} w\right)}{4 p_{2}^{2}} \tau r c d w_{1}, \\
d \rho(\mathrm{t})= & \frac{p_{2}^{2}+p_{1}^{2} w}{p_{2}^{2}} \varepsilon\left(\frac{3}{8} b \tau\left(p_{11}^{2} p_{12}+p_{12}^{3}\right) \beta_{0}^{2}-\frac{1}{2} q_{12} \mu+\frac{p_{2}^{2}+p_{1}^{2} w}{4 p_{2}^{2}} \tau^{2}\left(g_{11}^{2}+g_{12}^{2}\right) c^{2}\right) d t \\
& +\varepsilon^{1 / 2} \frac{\sqrt{2\left(g_{11}^{2}+3 g_{12}^{2}\right)}\left(p_{2}^{2}+p_{1}^{2} w\right)}{4 p_{2}^{2}} \tau c d w_{1} .
\end{aligned}
$$

Sample stability of the stochastic dynamical system is determined by the qualitative evaluation of the Lyapunov exponents. According to the multiplicative ergodic theorem [28], the top Lyapunov exponent $\lambda$ of the amplitude process is obtained as

$$
\lambda=\frac{p_{2}^{2}+p_{1}^{2} w}{p_{2}^{2}} \varepsilon\left[\frac{3}{8} b \tau\left(p_{11}^{2} p_{12}+p_{12}^{3}\right) E\left[\beta_{0}^{2}\right]-\frac{1}{2} q_{12} \mu+\frac{p_{2}^{2}+p_{1}^{2} w}{4 p_{2}^{2}} \tau^{2}\left(g_{11}^{2}+g_{12}^{2}\right) c^{2}\right]
$$

where $E\left[\beta_{0}^{2}\right]$ denotes the expected value of $\beta_{0}^{2}$. For the trivial solution $\beta_{0}^{2}=0$, (3.23) gives $\lambda=-(1 / 2) q_{12} \mu+\left(\left(p_{2}^{2}+p_{1}^{2} w\right) / 4 p_{2}^{2}\right) \tau^{2}\left(g_{11}^{2}+g_{12}^{2}\right) c^{2}=0$. Thus, the trivial solution is asymptotically stable with probability one (w.p.1) if $\lambda<0$, that is, $\mu<\left(g_{11}^{2}+g_{12}^{2}\right) \tau^{2} c^{2}\left(\left(p_{2}^{2}+p_{1}^{2} w\right) / 2 p_{2}^{2} q_{12}\right)$ and $q_{12}>0$ and unstable when $q_{12}<0$. 


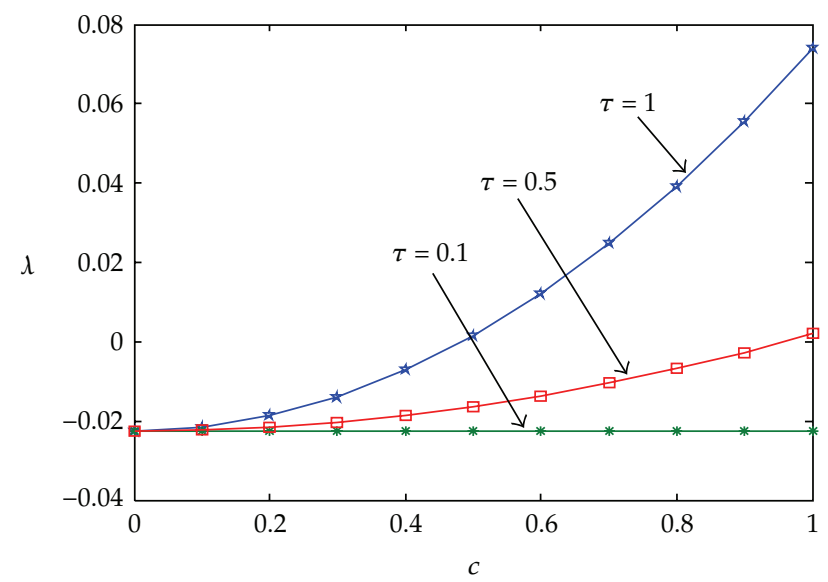

Figure 2: The top Lyapunov exponent $\lambda$ max of system (2.1) versus noise intensity for different values of time delay, $\tau \cdot a=1, \quad b=-2, \quad \varepsilon=0.1, \quad \mu=0.1$.

From Figure 2, we can observe that the top Lyapunov exponent $\lambda$ increases as the excitation intensity $c$ or time delay $\tau$ increases. The original system is stable if $\lambda<0$ and unstable if $\lambda>0$. When $\tau=1.0$, the system (2.1) becomes unstable at $c=0.447214$. The effect of the strength of noise on the human quiet standing losing stability is weak when time delay $\tau=0.10$. Obviously, the systems (2.1) is easily unstable if time delay $\tau$ is larger for the fixed excitation intensity $c$. A small change in the strength of noise may destabilize the quiet standing for a large delay.

\section{Conclusions}

The primary purpose of this paper is to study effects of delay and noise on asymptotic stability of the human quiet standing system with stochastic excitation. For the deterministic quiet standing system, its stable conditions, namely, the Hopf bifurcation, are presented and the regions of stability and instability are discussed. On one hand, by using proper variable transform, the second model for the human standing is changed into a first one. The given relation expressions between coefficients and physical quantities are practically significant too. On the other hand, the method of the center manifold reduction is generalized to investigate the asymptotic stability when the quiet standing system is subjected to a stochastic perturbation or the white noise. Compared to numerical methods, this analytical method permits some general conclusions for the classes of feedback functions. The necessary and sufficient conditions of the stability are obtained approximately by computing the largest Lyapunov exponent of the linearized stochastic ordinary differential system. The relation among the delay, the strength of noise, and the top Lyapunov exponent is represented in the parameter figure. The results show that the exponent is related not only to the strength of noise but also to the delay, namely, the reaction speed of brain, but such relation is not linear. In fact, the effect of the strength of noise on the human quiet standing losing stability is weak for a small delay. With the delay increasing, such effect becomes stronger and stronger. A small change in the strength of noise may destabilize the quiet standing for a large delay. It implies that a person with slow reaction easily lose the stability of his/her quiet standing. This conclusion is in agreement with observation in the real life. 
This may be of great help in diagnosing and treating some disease in this paper. However, noise is not always bad for cognitive performance, and moderate noise is beneficial for cognitive performance. Recently, it has been shown that noise can enhance human body balance via a mechanism known as stochastic resonance (SR) [29-31]. In the future, we will demonstrate the facilitating effects of SR by qualitative mathematical method.

\section{Appendix}

\section{A. The Computation of the $B$ and $\bar{\Psi}(0)$}

The values of the basis $\Phi(\theta) \in C$ for $P$ is given by

$$
\begin{aligned}
\phi(\theta) & =\Phi(\theta) \beta=\left[\phi_{1}(\theta), \phi_{2}(\theta)\right]=\alpha_{1} e^{i w \theta}+\alpha_{2} e^{-i w \theta} \\
& =\left[\left(\alpha_{1}+\alpha_{2}\right) \cos w \theta, i\left(\alpha_{1}-\alpha_{2}\right) \sin w \theta\right]=[\cos w \theta,-\sin w \theta]\left[\begin{array}{l}
\beta_{1} \\
\beta_{2}
\end{array}\right],
\end{aligned}
$$

where

$$
\alpha_{1}=p+i q, \quad \alpha_{2}=p-i q, \quad \alpha_{1}+\alpha_{2}=2 p=\beta_{1}, \quad i\left(\alpha_{1}-\alpha_{2}\right)=-2 q=\beta_{2} .
$$

Similarly, the basis $\psi(s) \in \widehat{C}$ for $\widehat{P}$ is of the form

$$
\Psi(s)=\left(\begin{array}{l}
\psi_{1}(s) \\
\psi_{2}(s)
\end{array}\right)=\left(\begin{array}{c}
\cos w s \\
-\sin w s
\end{array}\right), \quad 0 \leq s \leq 1
$$

The inner product matrix $(\psi(s), \phi(\theta))=\left(\psi_{j}(s), \phi_{k}(\theta)\right), i, j=1,2$ is given by

$$
(\psi(s), \phi(\theta))=\left(\begin{array}{cc}
\cos w s \cos w \theta & -\cos w s \sin w \theta \\
\sin w s \cos w \theta & \sin w s \sin w \theta
\end{array}\right) .
$$

The elements $\left(\psi_{j}(s), \phi_{k}(\theta)\right)$ substitute into the bilinear relation (3.11), which gives the nonsingular matrix

$$
(\Psi, \Phi)_{n s g}=\left(\begin{array}{ll}
\psi_{11} & \psi_{12} \\
\psi_{21} & \psi_{22}
\end{array}\right),
$$


where

$$
\begin{aligned}
& \psi_{11}=1+b \tau_{0} \int_{-1}^{0} \cos w(\xi+1) \cos w \xi d \xi=1+\frac{b \tau_{0}}{2}\left(\frac{1}{w} \sin w+\cos w\right), \\
& \psi_{12}=-b \tau_{0} \int_{-1}^{0} \cos w(\xi+1)(\sin w \xi) d \xi=\frac{b \tau_{0}}{2} \sin w, \\
& \psi_{21}=-b \tau_{0} \int_{-1}^{0} \sin w(\xi+\tau) \cos w \xi d \xi=-\frac{b}{2} \sin w \\
& \psi_{22}=b \tau_{0} \int_{-1}^{0} \sin w(\xi+1) \sin w \xi d \xi=\frac{b \tau_{0}}{2}\left(-\frac{1}{w} \sin w+\cos w\right) .
\end{aligned}
$$

Then, the basis $\Psi(s) \in \widehat{P}$ in $\widehat{C}$ of the adjoint (3.9) is normalized to $\bar{\Psi}(s)=\left[\bar{\psi}_{1}(s), \bar{\psi}_{2}(s)\right]^{T} \in \widehat{C}$. By computing

$$
(\Psi, \Phi)_{n s g}^{-1}=\frac{1}{\operatorname{det}(\Psi, \Phi)_{n s g}}\left(\begin{array}{cc}
\psi_{22} & -\psi_{21} \\
-\psi_{12} & \psi_{11}
\end{array}\right)=\frac{1}{\psi_{11} \psi_{22}-\psi_{12}^{2}}\left(\begin{array}{cc}
\psi_{22} & -\psi_{21} \\
-\psi_{12} & \psi_{11}
\end{array}\right)
$$

we obtain

$$
\bar{\Psi}(s)=(\Psi, \Phi)_{n s g}^{-1} \Psi(s)=\frac{1}{\psi_{11} \psi_{22}-\psi_{12}^{2}}\left(\begin{array}{cc}
\psi_{22} & -\psi_{21} \\
-\psi_{12} & \psi_{11}
\end{array}\right)\left(\begin{array}{l}
\psi_{1}(s) \\
\psi_{2}(s)
\end{array}\right)=\left(\begin{array}{l}
\bar{\psi}_{1}(s) \\
\bar{\psi}_{2}(s)
\end{array}\right)
$$

where

$$
\begin{aligned}
& \bar{\psi}_{1}(s)=\frac{1}{\psi_{11} \psi_{22}-\psi_{12}^{2}}\left(\psi_{22} \cos w s-\psi_{21} \sin w s\right) \\
& \bar{\psi}_{2}(s)=\frac{1}{\psi_{11} \psi_{22}-\psi_{12}^{2}}\left(-\psi_{12} \cos w s-\psi_{11} \sin w s\right) .
\end{aligned}
$$

Then, we have

$$
\bar{\Psi}(0)=\frac{1}{\psi_{11} \psi_{22}-\psi_{12}^{2}}\left(\begin{array}{c}
\psi_{22} \\
-\psi_{12}
\end{array}\right)
$$

The substitution of the elements $\left(\bar{\psi}_{j}(s), \phi_{\mathrm{k}}(\theta)\right), j, k=1,2$ into the bilinear relation (3.11) will yield the $2 \times 2$ identity matrix, namely,

$$
(\Psi, \Phi)_{i d}=\frac{1}{\psi_{11} \psi_{22}-\psi_{12}^{2}}\left(\begin{array}{cc}
\psi_{11} \psi_{22}-\psi_{12}^{2} & 0 \\
0 & \psi_{11} \psi_{22}-\psi_{12}^{2}
\end{array}\right)=\left(\begin{array}{ll}
1 & 0 \\
0 & 1
\end{array}\right)
$$


Defining the constant matrix $B \in C, \widehat{B} \in \widehat{C}$, the elements of $B, \widehat{B}$ at Hopf bifurcation are $B \equiv \widehat{B}=\left(\begin{array}{cc}0 & -w \\ w & 0\end{array}\right)$, which satisfy $A(\mu) \Phi(\theta)=\Phi(\theta) B, \widehat{A}(\mu) \Psi(s)=\Psi(s) \widehat{B}$. On the basis of algebraic simplifications

$$
e^{B \theta}=I+B \theta+\frac{(B \theta)^{2}}{2 !}+\frac{(B \theta)^{3}}{3 !}+\cdots=\left(\begin{array}{cc}
\cos w \theta & -\sin w \theta \\
\sin w \theta & \cos w \theta
\end{array}\right)
$$

the following formulas can be easily obtained

$$
\begin{aligned}
& \Phi(\theta)=\Phi(0) e^{B \theta}=(\cos w \theta-\sin w \theta), \quad-1 \leq \theta \leq 0, \\
& \Psi(s)=\Psi(0) e^{-\widehat{B} S}=(\cos w \theta-\sin w \theta)^{\mathrm{T}}, \quad 0 \leq s \leq 1 .
\end{aligned}
$$

\section{Acknowledgment}

This paper is supported by the State Key Program of National Natural Science of China under Grant no. 11032009 and the National Science Foundation for Distinguished Young Scholars of China under Grant no. 10625211.

\section{References}

[1] U. an der Heiden, "Delays in physiological systems," Journal of Mathematical Biology, vol. 8, no. 4, pp. 345-364, 1979.

[2] G. Stepan, "Delay effects in brain dynamics," Philosophical Transactions of the Royal Society A: Mathematical, Physical and Engineering Sciences, vol. 367, no. 1891, pp. 1059-1062, 2009.

[3] J. J. Collins and C. J. de Luca, "Open-loop and closed-loop control of posture: a random-walk analysis of center-of-pressure trajectories," Experimental Brain Research, vol. 95, no. 2, pp. 308-318, 1993.

[4] J. L. Cabrera and J. G. Milton, "On-off intermittency in a human balancing task," Physical Review Letters, vol. 89, no. 15, Article ID 158702, 4 pages, 2002.

[5] G. Haller and G. Stépán, "Micro-chaos in digital control," Journal of Nonlinear Science, vol. 6, no. 5, pp. 415-448, 1996.

[6] E. Enikov and G. Stépán, "Microchaotic motion of digitally controlled machines," Journal of Vibration and Control, vol. 4, no. 4, pp. 427-443, 1998.

[7] G. Stépán and L. Kollár, "Balancing with reflex delay," Mathematical and Computer Modelling, vol. 31, no. 4-5, pp. 199-205, 2000.

[8] A. J. van Soest and L. A. Rozendaal, "The inverted pendulum model of bipedal standing cannot be stabilized through direct feedback of force and contractile element length and velocity at realistic series elastic element stiffness," Biological Cybernetics, vol. 99, no. 1, pp. 29-41, 2008.

[9] C. W. Eurich and J. G. Milton, "Noise-induced transitions in human postural sway," Physical Review E, vol. 54, no. 6, pp. 6681-6684, 1996.

[10] H. Nakata and K. Yabe, "Automatic postural response systems in individuals with congenital total blindness," Gait and Posture, vol. 14, no. 1, pp. 36-43, 2001.

[11] J. Milton, J. L. Cabrera, T. Ohira et al., “The time-delayed inverted pendulum: implications for human balance control," Chaos, vol. 19, no. 2, Article ID 026110, 12 pages, 2009.

[12] B. W. Verdaasdonk, H. F. J. M. Koopman, S. A. van Gils, and F. C. T. van der Helm, "Bifurcation and stability analysis in musculoskeletal systems: a study in human stance," Biological Cybernetics, vol. 91, no. 1, pp. 48-62, 2004.

[13] A. Bottaro, Y. Yasutake, T. Nomura, M. Casadio, and P. Morasso, "Bounded stability of the quiet standing posture: an intermittent control model," Human Movement Science, vol. 27, no. 3, pp. 473495, 2008.

[14] F. Moss and J. G. Milton, "Medical technology: balancing the unbalanced," Nature, vol. 425, no. 6961, pp. 911-912, 2003. 
[15] T. Hosaka, T. Ohira, C. Luciani, J. L. Cabrera, and J. G. Milton, "Balancing with noise and delay," Progress of Theoretical Physics Supplement, vol. 161, pp. 314-319, 2006.

[16] K. Masani, A. H. Vette, N. Kawashima, and M. R. Popovic, "Neuromusculoskeletal torque-generation process has a large destabilizing effect on the control mechanism of quiet standing," Journal of Neurophysiology, vol. 100, no. 3, pp. 1465-1475, 2008.

[17] Y. Asai, Y. Tasaka, K. Nomura, T. Nomura, M. Casadio, and P. Morasso, “A model of postural control in quiet standing: robust compensation of delay-induced instability using intermittent activation of feedback control," PLoS ONE, vol. 4, no. 7, article e6169, 2009.

[18] W. Yao, P. Yu, and C. Essex, "Delayed stochastic differential model for quiet standing," Physical Review E, vol. 63, no. 2, Article ID 021902, 8 pages, 2001.

[19] J. Boulet, R. Balasubramaniam, A. Daffertshofer, and A. Longtin, "Stochastic two-delay differential model of delayed visual feedback effects on postural dynamics," Philosophical Transactions of the Royal Society A: Mathematical, Physical and Engineering Sciences, vol. 368, no. 1911, pp. 423-438, 2010.

[20] J. Milton, J. L. Townsend, M. A. King, and T. Ohira, "Balancing with positive feedback: the case for discontinuous control," Philosophical Transactions of the Royal Society A: Mathematical, Physical and Engineering Sciences, vol. 367, no. 1891, pp. 1181-1193, 2009.

[21] G. Stepan, "Delay effects in the human sensory system during balancing," Philosophical Transactions of the Royal Society A: Mathematical, Physical and Engineering Sciences, vol. 367, no. 1891, pp. 1195-1212, 2009.

[22] M. Grigoriu, "Control of time delay linear systems with Gaussian white noise," Probabilistic Engineering Mechanics, vol. 12, no. 2, pp. 89-96, 1997.

[23] Z. H. Liu and W. Q. Zhu, "Asymptotic Lyapunov stability with probability one of quasi-integrable Hamiltonian systems with delayed feedback control," Automatica, vol. 44, no. 7, pp. 1923-1928, 2008.

[24] L. Arnold and P. Boxler, "Eigenvalues, bifurcation, and center manifolds in the presence of noise," in Differential Equations (Xanthi, 1987), C. M. Dafermos, G. Ladas, and G. Papanicolaou, Eds., vol. 118 of Lecture Notes in Pure and Applied Mathematics, pp. 33-48, Dekker, New York, NY, USA, 1989.

[25] J. Hale, Theory of Functional Differential Equations, Springer, New York, NY, USA, 2nd edition, 1977.

[26] G. Stepan, Retarded Dynamical Systems, Longman, Harlow, UK, 1989.

[27] R. L. Stratonovich, "On stochastic processes defined by differential equations with small parameter," Theoretical Probability, pp. 390-406, 1966.

[28] Y. I. Oseledec, "A multiplicative ergodic theorem: Lyapunov characteristic number for dynamical systems," Transactions of the Moscow Mathematical Society, vol. 19, pp. 197-231, 1968.

[29] S. Guillouzic, I. L'Heureux, and A. Longtin, "Small delay approximation of stochastic delay differential equations," Physical Review E, vol. 59, no. 4, pp. 3970-3982, 1999.

[30] D. C. Gravelle, C. A. Laughton, N. T. Dhruv et al., "Noise-enhanced balance control in older adults," NeuroReport, vol. 13, no. 15, pp. 1853-1856, 2002.

[31] A. A. Priplata, B. L. Patritti, J. B. Niemi et al., "Noise-enhanced balance control in patients with diabetes and patients with stroke," Annals of Neurology, vol. 59, no. 1, pp. 4-12, 2006. 


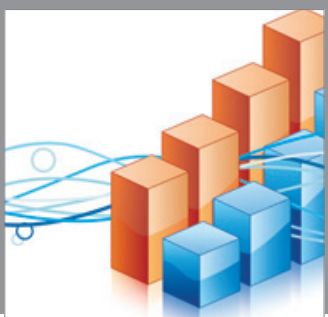

Advances in

Operations Research

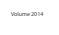

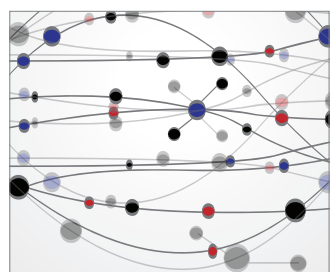

\section{The Scientific} World Journal
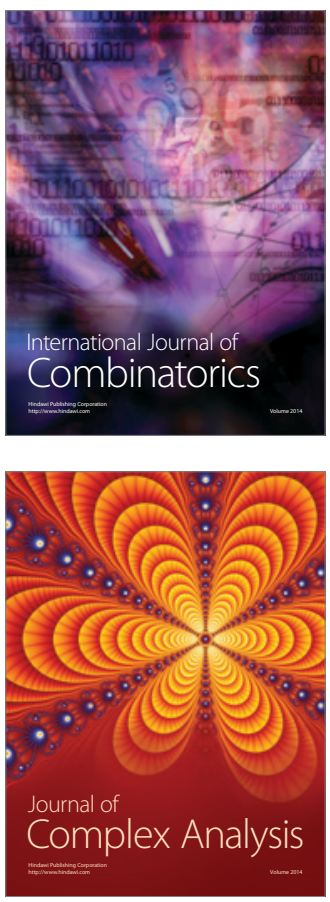

International Journal of

Mathematics and

Mathematical

Sciences
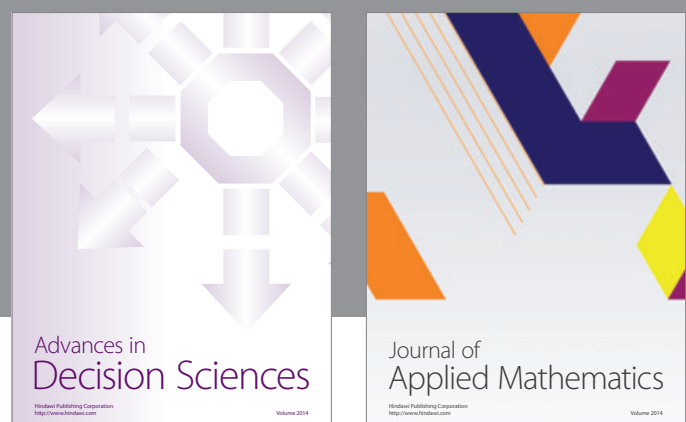

Journal of

Applied Mathematics
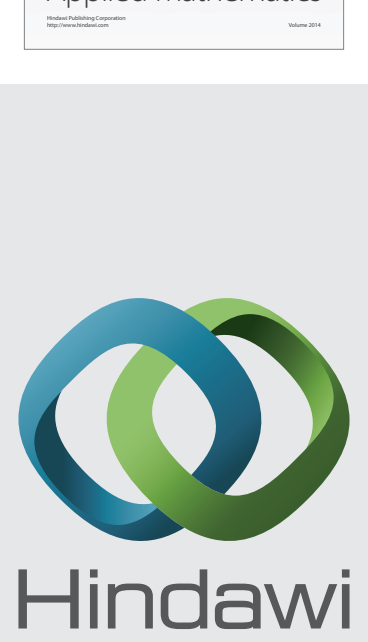

Submit your manuscripts at http://www.hindawi.com
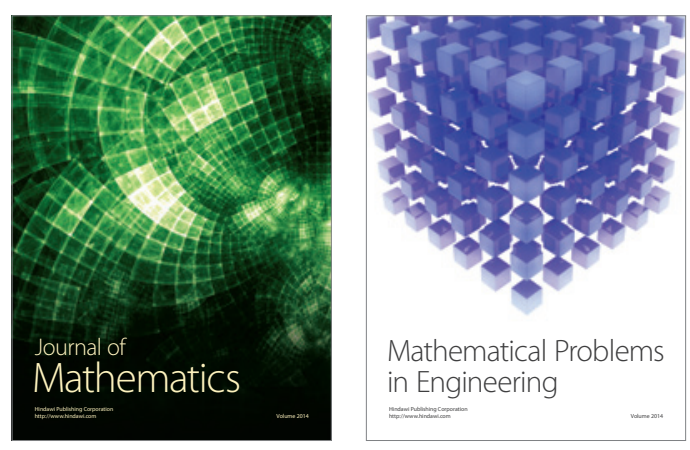

Mathematical Problems in Engineering
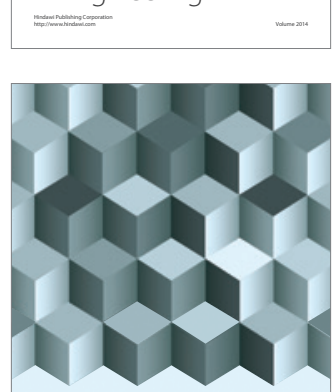

Journal of

Function Spaces
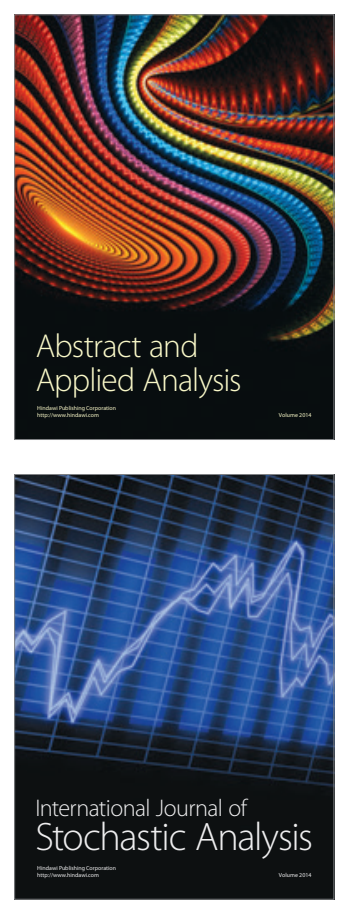

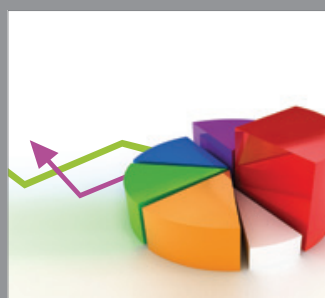

ournal of

Probability and Statistics

Promensencen
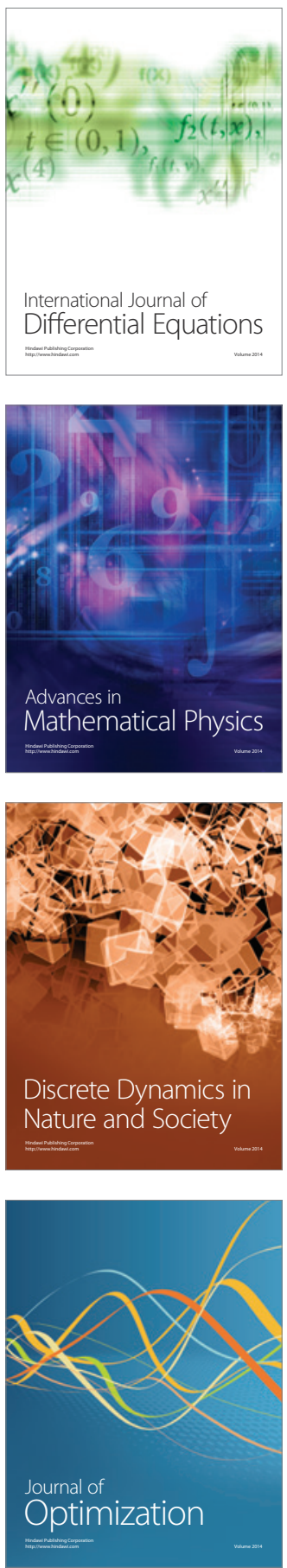